\title{
SINGLE MOLECULES AND SINGLE NANOPARTICLES AS WINDOWS TO THE NANOSCALE
}

\author{
nartín Caldarola and Michel Orrit - DOI: https://doi.org/10.1051/epn/2018201 \\ - Single-Molecule Optics, Huygens-Kamerlingh Onnes Laboratory, University Leiden - Niels Bohrweg 2, 2300 RA Leiden (Netherlands)
}

\begin{abstract}
Since the first optical detection of single molecules, they have been used as nanometersized optical sensors to explore the physical properties of materials and light-matter interaction at the nanoscale. Understanding nanoscale properties of materials is fundamental for the development of new technology that requires precise control of atoms and molecules when the quantum nature of matter cannot be ignored. In the following lines, we illustrate this journey into nanoscience with some experiments from our group.
\end{abstract}

\begin{abstract}
General background
According to Richard Feynman, the single most important insight science brought us is that "matter is made of atoms". Throughout the $20^{\text {th }}$ century, we have grown accustomed to more and more direct atomic and molecular manifestations, starting with Brownian motion and X-ray diffraction, and eventually ending with electron micrographs showing single atoms and molecules, or scanning tunneling microscopy images, which routinely allow us to 'feel' individual atoms and molecules on solid surfaces in an immediate way. But what about'seeing' atoms and molecules with light? This century-old dream was born with fluorescence experiments by Jean Perrin [1] around 1910, but only came true in the 1970s with attenuated atomic beams and later with single trapped ions in ultrahigh vacuum. For molecules in condensed matter, the limit of optical single-molecule detection was reached only in the late 1980s, essentially because of two technical problems: background emission from the molecule's surroundings and limited total number of fluorescence photons for each molecule because of bleaching, i.e., photo-induced degradation of the emitter. Both problems were solved by cryogenic experiments at liquid-helium temperatures, through resonant absorption cross sections and thanks to the excellent photostability of molecules held in a rigid cage. These cryogenic experiments gave rise to many striking observations in nanophysics and quantum optics. However, single-molecule optics only turned into a real revolution with ambient conditions. The loss of a factor 100,000 in cross section upon warming from
\end{abstract}

\section{Single-molecule experiments are superb testing grounds for quantum optics and nanoscience $\square$}

liquid-helium to room temperature is compensated by an equivalent improvement in optical quality and selectivity by the diffraction-limited spots of modern microscopes. Thanks to objective lenses with large numerical apertures, single-molecule techniques became readily applicable in material science and biology.

A typical single-molecule fluorescence experiment at room temperature requires a confocal microscope, laser excitation of a small, diffraction-limited volume of some hundreds of nm in size, and a photon-counting detection of the Stokes-shifted fluorescence emitted by the excited spot, often collected by the excitation objective lens. The Stokes shift allows for easy and efficient spectral filtering of the signal. Critical points for a high signal-to-background ratio are a tiny (diffraction-limited) excitation volume, clean samples and optics, and a high stability of setup, laser, and sample. With this basic setup, a large number of time- and space- resolved experiments can be conducted on nanometer scales. In this article, we would like to share our enthusiasm for the novel physics that the signals of single molecules have opened and illustrate them with some work from our group.

\section{Single molecules as single-photon sources and nanoprobes}

Cryogenic single-molecule experiments are superb testing grounds for quantum optics and for nanoscience. A single molecule's emission on its lifetime-limited zero-phonon line (a purely electronic molecular transition with no phonons involved) provides indistinguishable photons one at a time, in a so-called Fock state of the electromagnetic field, i.e. a state with a well-defined number of photons. These states can be used to perform fundamental tests on quantum mechanics, as done in the group of R. Hanson [2]. Moreover, single molecules can be used to probe the 
local properties of the surrounding material, since they are extremely small and highly sensitive to their nano-environment. For example, one may exploit the high sensitivity of a single-molecule spectral line to its environment to detect a mechanical deformation of the crystal embedding the molecule [3]. In a recently started project, we are trying to detect single charges in a metal island through the Stark effect, i.e., the shift of the molecule's transition by the charge's electric field. This optical access to charge would be complementary to standard current measurements and will give researchers new insight into the workings of electronic components, and help them design new methods for information storage and transfer.

Molecular fluorescence is generally polarized along one of the molecular axes. By following this polarization as a function of time for individual molecules, one can explore the rotational diffusion of the molecules, and thereby the local rheological properties of their environment at nanometer scales. We have studied this rotational diffusion in a molecular glass former, glycerol, whose viscosity increases by more than 10 orders of magnitude between room temperature and the glass transition, at about $200 \mathrm{~K}$. The first unexpected result was that single dye molecules at different positions in the material, although chemically identical, were tumbling at widely different rates. These differences reveal changes in local viscosity, which must be related to changes in local structure. A second surprise was that for samples kept a long time at low temperature, the viscosity fluctuations did not relax even over days or longer, whereas the molecular relaxation times were on the order of seconds [4]. Figure 1 shows examples of confocal images of single molecules in a glass matrix, where the polarization of the emitted light is split to infer the molecular rotation dynamics of the molecules. More recent work with larger particles, gold nanorods, has confirmed this dynamical heterogeneity on even larger length and time scales. The picture of a glass former conveyed by single molecules is that of a strongly disordered medium, in which local density fluctuations lead to large fluctuations of viscosity. Due to the large viscosity, these heterogeneities cannot relax on the time scale of the experiments, leading to very long memory times of the viscosity fluctuations.

The latter two examples illustrate how optically detected single molecules can be used to extract local information about a medium at nanometer scales. Consistently, observations at molecular scales unveil a surprisingly large heterogeneity and much larger disorder than was expected from conventional ensemble experiments.

\section{Plasmonics: single-molecule sensing and enhanced light-matter interactions}

Small metal nanoparticles (smaller than $100 \mathrm{~nm}$ ) present a very strong optical mode called plasmon, in which conduction electrons oscillate collectively around the heavy, nearly immobile ions. This mode, which is

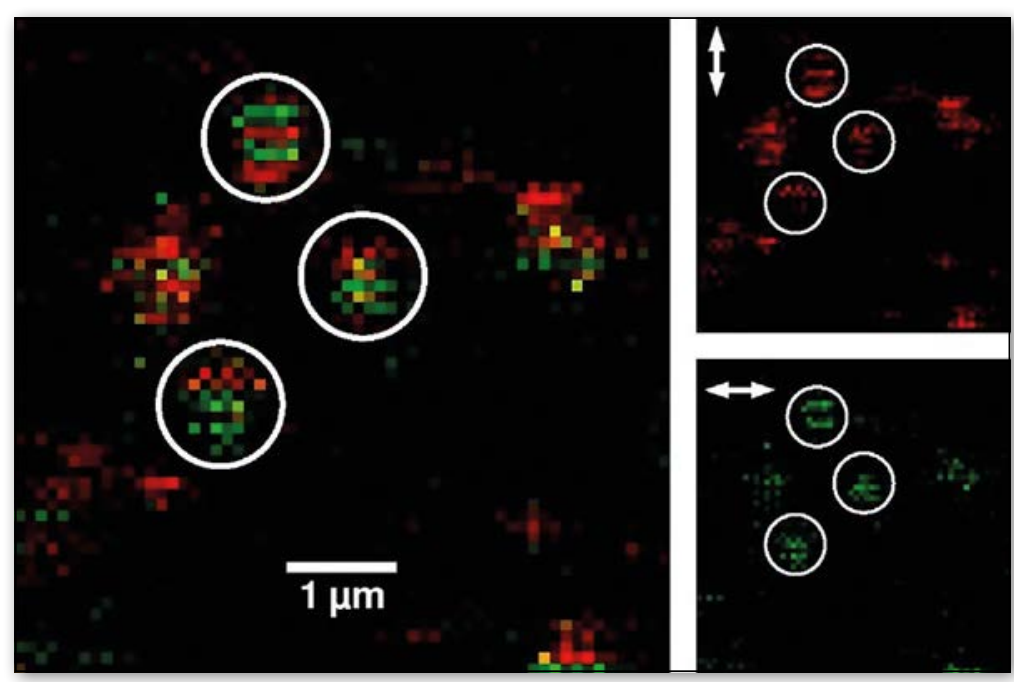

responsible for the specific optical properties and for the strong colors of metal suspensions in water, can be tuned by changing the aspect ratio of the particles during synthesis. For example, gold nanorods can be synthetized with aspect ratios ranging between 1 (spheres, plasmon at $530 \mathrm{~nm}$ ) and 5 or longer (rods, plasmons at $900 \mathrm{~nm}$ or longer). The large surface charge densities developed in plasmon oscillations lead, via Gauss' theorem, to highly localized oscillating electric fields, which are much higher than the field of the incoming excitation wave. Moreover, the field is extremely confined in a tiny volume around the tips of the particle. This enhanced and highly localized field can be useful for many applications of light-matter interactions, and we have explored two examples with gold nanorods: plasmonic sensing of non-absorbing protein molecules and single-molecule fluorescence enhancement. The former example is based on the detection of the plasmon spectral shift due to the binding of a single protein, some nanometers in size, at the tip of a nanorod [5]. The latter one relies on the enhanced excitation of a fluorescent molecule diffusing or fixed near the tip of the nanorod. In addition to the excitation enhancement (which can be as high as $\mathbf{3 0 0}$ for a gold nanorod), the emission of the dye is enhanced because the gold nanoparticle acts as a resonant antenna, improving the coupling of the molecular dipole with the propagating optical wave, leading to an increase by about 10 times in the emission. In this way, the fluorescence intensity of a molecule in the near field can be enhanced more than 1,000 times compared to the emission of the same molecule away from the gold nanorod [6].

Recently we took the next step in fluorescence enhancement and used it to tackle another problem: study a single molecule's electrochemical properties optically [7]. We successfully combined the electrochemical control of the oxidation state of a redox sensitive dye, methylene blue (MB), with an enhanced-fluorescence readout. This dye has the interesting property of being absorbing but $\triangle$ FIG. 1: Single molecules as probes for local rheological properties. (left) Single PDI molecules in glycerol at a temperature of 209.6 K. The red and green images correspond to the two orthogonal polarization channels, shown separately on the right. Extracted from [4]. 


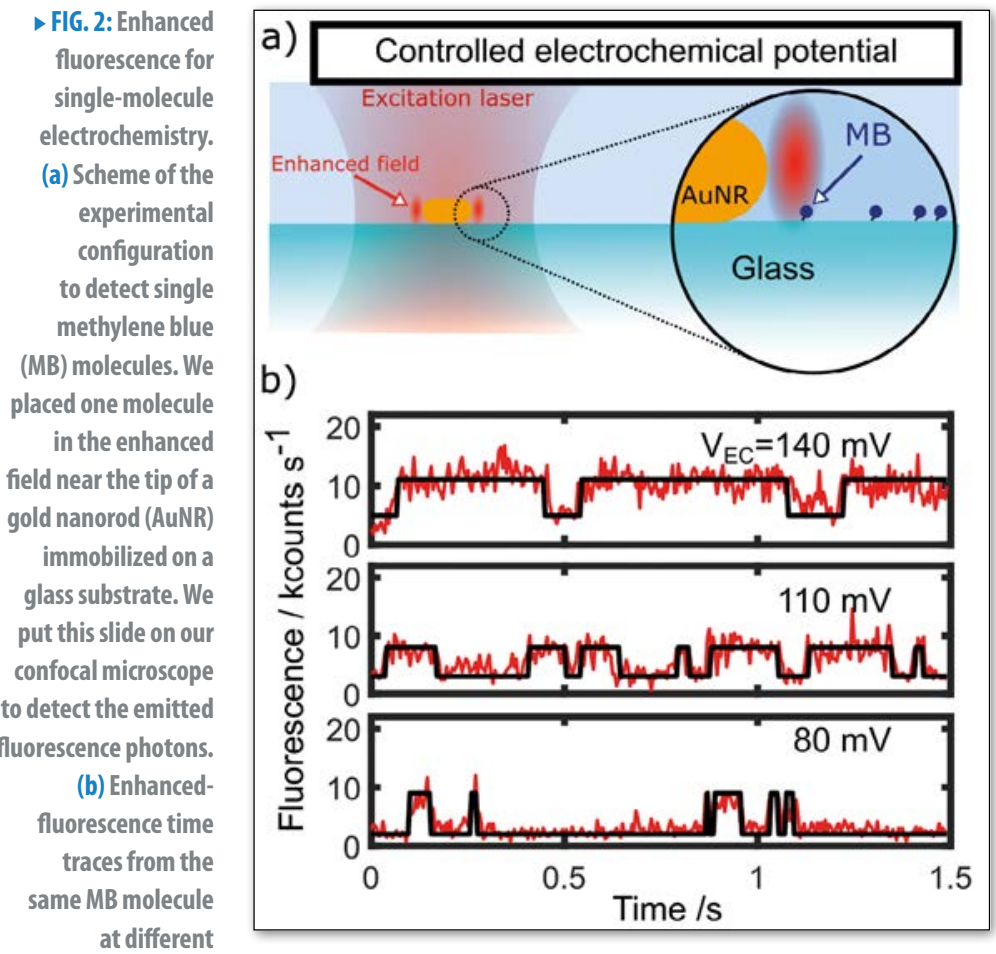

weakly fluorescent in the oxidized state and non-absorbing in the reduced state. Thus, it can be used as a redox indicator. The main limitation for this application is the dye's very low fluorescence quantum yield. However, we overcame this limitation by enhancing the fluorescence with a gold nanorod, thus reaching single-molecule sensitivity. Figure 2 a) shows the schematic representation of the experiment where we externally control the electrochemical potential and simultaneously detect $\mathrm{MB}$ molecules optically. Figure $2 \mathrm{~b}$ ) shows the enhanced-fluorescence time trace of the same single MB molecule at different electrochemical conditions. These plots demonstrate that the blinking cycles are redox-induced, and provide the mid-point potential of the molecule.

The mentioned examples show how metallic nanoparticles can be used as bridges between molecular sizes and the optical wavelength. By enhancing light-matter interaction, plasmonics enable the detection of biological species and reveal their chemical properties at the single-molecule level.

\section{Magnetic properties of gold nanorods}

Nanoparticles can be also used as nanoprobes to gain knowledge about the properties of matter at the nanoscale. Although metal nanoparticles have been studied extensively, they still present some intriguing properties

\section{Resonant metallic nanoparticles can be used to bridge molecular sizes with optical wavelengths to enhance light-matter interactions and allow optical detection of single molecules $7 \rightarrow$}

that require detailed investigation. Studies of single particles would provide a better understanding of the physical mechanisms involved. In the course of our work on gold nanorods, we have explored their magnetic properties in the high-field magnet in Nijmegen [8]. Surprisingly, the nanorods showed preferential orientation along the magnetic field, much more so than was expected from the diamagnetic susceptibility of bulk gold metal. The observed orientation was consistent with giant magnetic dipole moments induced in the metal nanorod by the change of magnetic field. These currents do not relax, as the dimensions of the rods are much less than the mean free path of electrons in the metal. In the future, we plan experiments on single rods, in which theory predicts large variations of the amplitude of these persistent currents. These experiments would test the presence and strength of this intriguing effect in structures different from the metal loops investigated earlier by atomic force microscopy [9].

\section{Explosive dynamics of vapor nanobubbles}

Our recent study of nanobubble formation is another example of complex physics that can be accessed with single nanoparticles. Excitation of plasmons leads to currents in the metal and therefore to Joule dissipation. Thus, metallic nanoparticles have been extensively used as nanoheaters for different applications, including photothermal cancer therapy. The temperature increase can be several hundreds of degrees and thus it can be used to bring a liquid surrounding the particle to boiling. We try to understand how this nanoscale boiling differs from the one we are familiar with at macroscopic scales. One of the differences is due to surface tension because the bubble is very small (the gas in a 100-nm-radius bubble has a pressure of about $10 \mathrm{~atm}$ ). We find that the liquid can become superheated, leading to explosive boiling. This is a violent phenomenon, taking place in some tens of nanoseconds at 100-nanometer scales, which is accompanied by the release of sound waves. These waves can reflect on nearby interfaces and further interfere with the transient bubble [10]. Figure 3 a) shows the schematic design of our experiment for nanobubble generation and detection, while Figure 3 (b, c) presents measurements of the explosive behaviour of the system. We assign the observed quasi-periodic bubble explosions to relaxation oscillations of this highly nonlinear system, where optical energy is first stored in hot water layers close to the particle, before being released in a bubble explosion. On the one hand there are possible applications for the emitted sound waves, for example to kill cancer cells, on the other hand, it is intriguing to explore phase transitions on smaller and smaller scales, eventually testing the validity of the thermodynamic limit. 


\section{Conclusion}

The optical detection of single molecules and single nanoparticles opens up nearly non-invasive exploration of matter at nanometer scales. In this sense, we can say that those experiments open windows to the nanometer scale. Most experiments on single molecules and single nanoparticles reveal unexpected variety and heterogeneity in the physical and chemical properties of matter at the smallest scale, which up to now had been hidden in ensemble experiments. Such exquisite sensitivity is all-important for complex and heterogeneous systems, particularly biological ones. The development of embryos or tumors dramatically demonstrates how determining tiny numbers of molecules can be for the ultimate fate of a whole living organism. [

\section{About the Authors}

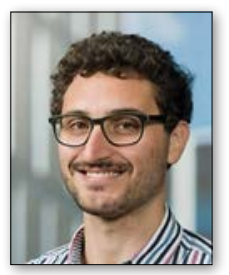

Martin Caldarola is a postdoctoral researcher at the Single-Molecule Optics group at Leiden University. He holds a $\mathrm{PhD}$ in physics from the University of Buenos Aires and his expertise is in the field of nanophotonics. His research interests include light-matter interactions, specifically the interaction between single molecules and nanostructures.

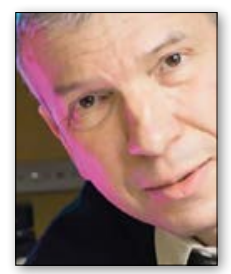

Michel Orrit's scientific field is the interaction of light with organic molecules in dense matter. His scientific breakthrough was the first observation of a single molecule using fluorescence. Orrit moved to Leiden in 2001, where his group applies single-molecule spectroscopy to molecular photophysics, solid-state dynamics, and nonlinear optics. His current interests include gold nanoparticles and molecules as nano-probes of structure and dynamics of soft condensed matter.

\section{References}

[1] J. Perrin, Atoms (transl. D. L. Hammick, 2nd Edition) New York, Van Nostrand 1923, p. 104.

[2] B. Hensen et al., Nature 526, 7575 (2015).

[3] Y. Tian, P. Navarro, M. Orrit, , Phys. Rev. Lett. 113, 13 (2014).

[4] R. Zondervan, Single-molecule dynamics at variable temperatures, PhD thesis at Leiden Institute of Physics, Faculty of Mathematics and Natural Sciences, Leiden University (2006).

[5] P. Zijlstra, P. M. Paulo, M. Orrit, Nature Nanotech. 7, 6 (2012).

[6] S. Khatua, P. M. Paulo, H. Yuan, A. Gupta, P. Zijlstra, M. Orrit, ACS Nano 8, 5 (2014).

[7] W. Zhang, M. Caldarola, B. Pradhan, M. Orrit, Ange. Chem. Int. Ed. 56, 13 (2017).

[8] P. G. Van Rhee, P. Zijlstra, T. G. A. Verhagen, J. Aarts, M. I. Katsnelson, J. C. Maan, M. Orrit, P. C. M. Christianen, Phys. Rev. Lett. 111, 12 (2013).

[9] A. C. Bleszynski-Jayich, W. E. Shanks, B. Peaudecerf, E. Ginossar, F. Von Oppen, L. Glazman, J. G. E. Harris, Science 326, 5950 (2009).

[10] L. Hou, M. Yorulmaz, N. R. Verhart, M. Orrit, New J. Phys. 17, 1 (2015).

\section{We can think of single molecules as windows to the nanoscale. Using their exquisite sensitivity to the local environment, we can unravel the mysteries of matter in the nanoscale $\because 7$}
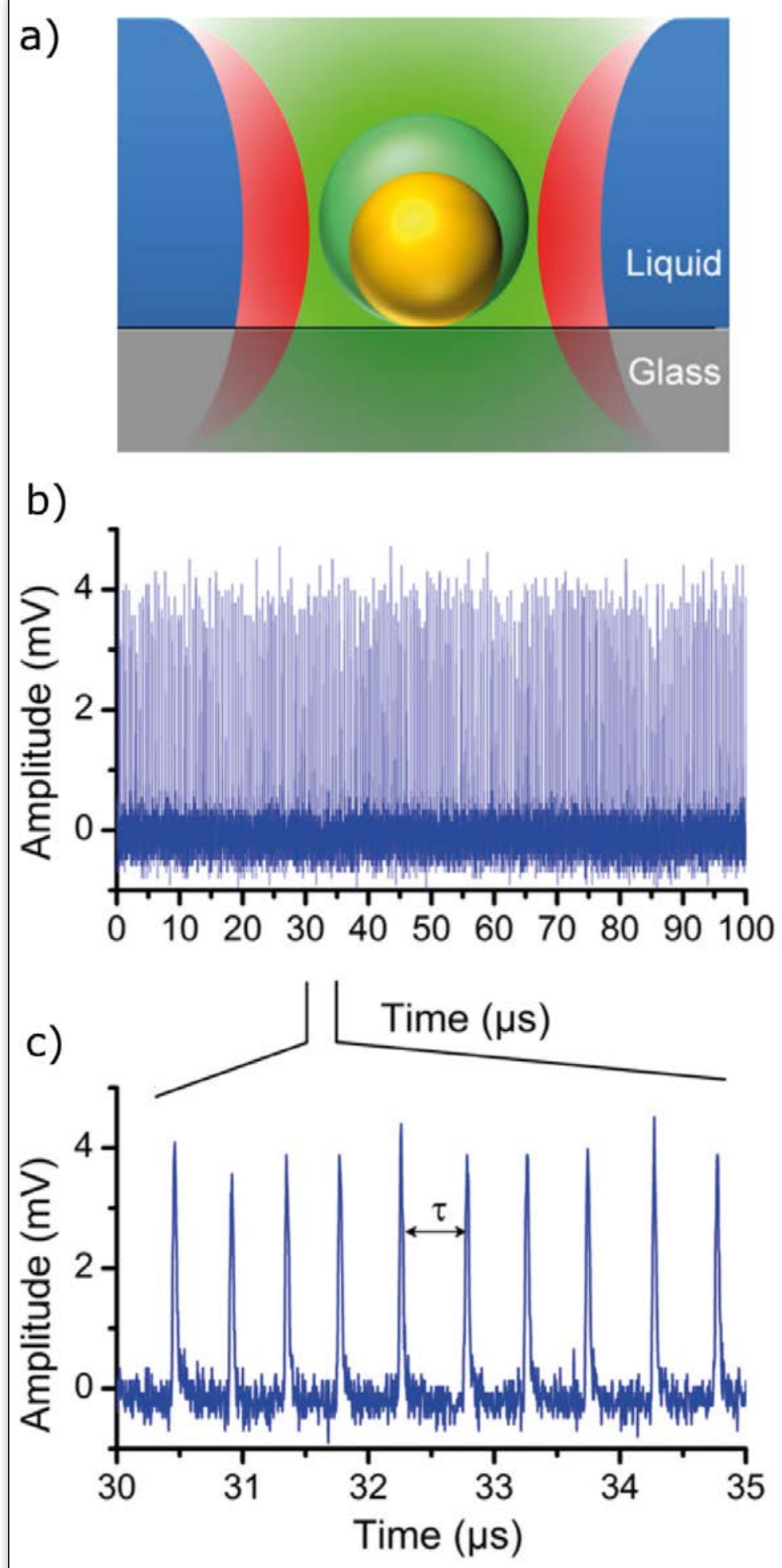

$\triangle$ FIG. 3: Nanobubble generation. (a) Scheme of the setup to create and probe nanobubbles. We use a focused green laser beam to heat the particle and a red one to detect the bubble. (b) Time trace of the scattered red light showing explosive events at the sub-microsecond time scale. (c) zoom of the time trace showing the quasi-periodicity of bubble formation. From Ref. [10]. 